\title{
Strategisches Management und Unternehmenskultur in Mittelmeerländern: Der Fall Spanien
}

\author{
S. G. Echevarria ${ }^{1}$
}

Prof. Dr. Dr. Santiago Garcia Echevarría ist ordentlicher Professor und Inhaber des Lehrstuhls für Unternehmungspolitik an der Universität Alcalà de Henares. Er durchlief seine wissenschaftliche Grundausbildung an der Universität Bilbao, wo er den Ausweis eines Intendente Mercantil erlangte und mit dem Lizenziat in Wirtschaftswissenschaften abschloß. Im Jahre 1962 promovierte er zum Dr. rer. pol. an der Universität Köln. Anschließend erlangte er das Doktorat in Wirtschafts- und Unternehmungswissenschaften an der Universidad Complutense in Madrid. Er erfüllte Lehraufträge an der Universidad de Deusto und an der Universidad Complutense. Im Jahre 1972 wurde er auf den Lehrstuhl für Wirtschaftspolitik der Unternehmung an der Universität Barcelona berufen. Zur Zeit ist er ordentlicher Professor der Universität Alcalà de Henares und Direktor des Instituts für Unternehmungsführung und Organisation.

Seine wichtigsten Veröffentlichungen sind die folgenden: „Wirtschaftsentwicklung Spaniens unter dem Einfluß

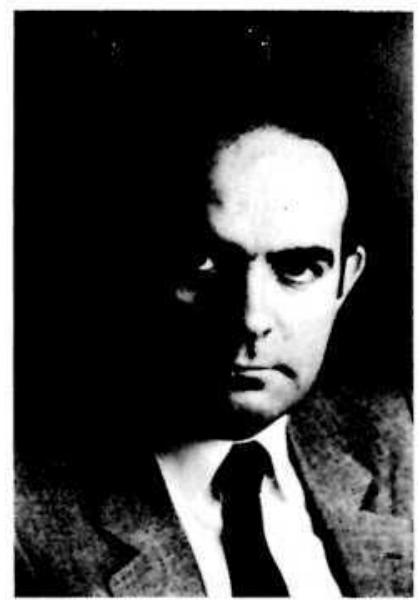
der Europäischen Integration" (1964); Planificación y Pronóstico en la Economía de la Empresa“ (1970), „Economía de la Empresa y Politica Economica de la Empresa“ (1972), „Introducción a le Economía de la Empresa“ (1974), „Politica Economica de la Empresa“ (1977), „Empressa y Orden Económico“ (1980), „Orden económico en una sociedad pluralista" (1983).

\section{Zusammenfassung}

Spanien weist in seiner Wirtschaftsentwicklung in der Gegenwart einen Rückstand von gut 15 Jahren im Vergleich zu den mitteleuropäischen Ländern auf. Der Industrialisierungsprozeß setzte im Jahre 1960 ein. Bis Mitte der 70er Jahre standen die technischen Prozesse ganz im Vordergrund. Es folgte dann eine Beachtung der Absatzprobleme. Die ganze Entwicklung erfolgte im Rahmen einer in hohem Maße gelenkten Wirtschaft, in welcher der Staat die Allokation der wirtschaftlichen Mittel vornahm und den Unternehmungen eine Abschirmung gab. Der Eintritt in die EG schafft für Spanien völlig neue Probleme. Diese können nur durch einen Übergang zu einer freien Marktwirtschaft bewältigt werden. Diese muß durch eine Neuausrichtung der Unternehmer und Führungskräfte getragen sein. An die Stelle einer auf Verwaltung beschränkten

\footnotetext{
${ }^{1}$ Professor Dr. Dr. Santiago Garcia Echevarria, Universidad de Alcalà de Henares, Spanien.
} 
Lenkung der Unternehmung, in welcher der Zeithorizont nur ein Jahr beträgt, wird eine strategische Ausrichtung treten müssen. Die fehlende unternehmerische Ausbildung muß nachgeholt werden, und es wird vor allem eine Dynamisierung der Unternehmungskultur erforderlich werden.

\section{Einführung}

Es hat praktisch sehr lange gedauert bis man erkannt hat, daß die Wirtschaftsfähigkeit eines Landes in einem sehr großen Maße von der Leistungsfähigkeit seiner Unternehmensführung abhängt, und dies besonders in unseren Ländern. Es besteht eine sehr enge Interdependenz zwischen der Leistungsfähigkeit der Unternehmensführung und der Wettbewerbsfähigkeit einer Wirtschaft, was wiederum in Krisenzeiten zum Ausdruck kommt.

Gerade die spanische Entwicklung ab 1960 bestätigt diese Interdependenz. Im Jahre 1960 begann der moderne Industrialisierungsproze $\beta$ der spanischen Wirtschaft, eine Entwicklung, die praktisch bis Mitte der 70er Jahre andauerte. In diesen zwei Jahrzehnten kann man sehr deutlich feststellen, wie die technischen Prozesse der Industrialisierung im Vordergrund stehen und wie Managementfragen kaum beachtet wurden.

In dieser entscheidenden Periode der Industrialisierung gab es kaum Bemühungen um die Einführung eines leistungsfähigen Managements. Die spanischen Unternehmen haben selten unter dem Druck der Kalkulation gestanden. Die Hauptursachen dieser Entwicklung sind meines Erachtens folgende:

1. Der wirtschaftspolitische Rahmen für die Unternehmenstätigkeit vermittelte eine ,sichere“ Umwelt innerhalb einer verwalteten Wirtschaftsordnung. Sowohl die Inputs wie die Outputs der Unternehmenstätigkeit wurden von der Öffentlichen Verwaltung mittelbar oder unmittelbar zu sehr günstigen und stabilen Bedingungen zur Verfügung gestellt. Die Planung der großen Investitionsprojekte basierte auf der Grundlage der damaligen stabilen Bedingungen. Die Erwartung eines permanenten Wachstums unter diesen günstigen Bedingungen war die Grundlage des Entscheidungsprozesses der Unternehmungen. Vor allem die finanziellen Voraussetzungen sowie die niedrigen Arbeitskosten und auch die Steuervorteile und Subventionen bildeten die bestimmenden Strukturmerkmale der spanischen Unternehmungsführung.

2. Der gesamte Industrialisierungsproze $\beta$ fand unter der Leitung und Verantwortung von technisch ausgebildeten Managern statt. Die Techniker, vor allem die besonderen Eliten aus den technischen Hochschulen, bildeten die spanische Unternehmensführung. Andere Managergruppen kamen aus der Staatsverwaltung, was hauptsächlich im Bereich des Personalwesens und der Verwaltung der Fall war. Die enge Beziehung zur öffentlichen Verwaltung war eine entscheidende Bindung für die Unternehmenstätigkeit.

3. Das gesamte Ausbildungssystem, vom Universitätsniveau bis zu den Berufsschulen, hat in diesen zwei Jahrzehnten keine entsprechende betriebswirtschaftliche Ausbildung angeboten. Dies dauerte praktisch bis Mitte der 70er Jahre an. Auch heute sind weiterhin die Ausbildungsstrukturen in bezug auf 
Managementlehre und betriebswirtschaftliche Ausbildung noch ungenügend und wirklichkeitsfremd.

Die Ergebnisse dieser Konstellation sind heute höhere Produktionskosten als Folge der niedrigen Produktivität der Führung und eine geringe internationale Wettbewerbsfähigkeit der spanischen Industrie, von Ausnahmefällen abgesehen.

In der Periode einer starken Wachstumsrate, im Rahmen einer protektionistischen und subventionsfreudigen Wirtschaftspolitik und in ständiger Begleitung einer beachtlichen Inflationsrate trat die große Lücke in der Managementstruktur nicht so auffällig hervor. Im Moment einer Wirtschaftskrise aber erscheint diese in ihrer ganzen Auswirkung. Diese Komponente stellt meines Erachtens den Hauptunterschied zwischen der gegenwärtigen westeuropäischen und der spanischen Wirtschaftskrise dar. Das gesellschaftliche Bewußtsein für wirtschaftliche Probleme und Problemlösungen ist in Spanien nur schwach entwickelt. Diese Situation betrifft nicht nur die spanischen Unternehmer sondern auch die Politiker, Gewerkschaftler, die öffentliche Verwaltung und das Ausbildungssystem. Sonst wäre es nicht möglich gewesen, da $ß$ in der gesellschaftspolitischen Übergangsperiode der letzten zehn Jahre die Wirtschaftsprobleme und die Unternehmensprobleme in den Hintergrund geschoben wurden. Die fehlende betriebswirtschaftliche Ausbildung ist einer der entscheidenden Faktoren für die relative Schwäche der spanischen Wirtschaft und der spanischen Unternehmungen.

Eine von uns vorgenommene empirische Erhebung (als erste dieser Art in Spanien) zeigt eine sehr deutliche Distanz zur Rolle der Unternehmer. Nur 16\% aller Befragten möchten eine Unternehmertätigkeit ausüben und nur $11 \%$ der Studenten möchten in der Zukunft als Manager tätig sein. Die Neigung der Studenten geht zur Beamtenlaufbahn.

Wenn wir den Industrialisierungsprozeß in Spanien analysieren, um den Rahmen für die Entwicklung der Unternehmensführung abzustecken, können wir folgendes feststellen: Nach einer Periode von 3 Jahrzehnten des Protektionismus wird Anfang der 60er Jahre eine marktwirtschaftliche Orientierung eingeführt, aber schon im Jahre 1964 wird nach französischem Muster eine indikative Planung als wirtschaftspolitische Grundlage verankert.

In den ersten Bemühungen, mehr Wettbewerb einzuführen, ersticken die Planungskonzeptionen die Herausforderung zu einer Modernisierung der Unternehmensführung. Die indikative Planung bedeutet hauptsächlich eine Stärkung der Wirtschafts-Verwaltung, was auf die Führungskompetenzen und -fähigkeiten zurückwirkt. Der fehlende Wettbewerb, die stabilen Verhältnisse der Umwelt und die abgesprochenen Tätigkeitsprogramme bieten keine Grundlage für eine angepaßte Entwicklung der Unternehmensführung. Dies dauerte bis Mitte der 70er Jahre an. Daran schloß sich die Stagnationsperiode der Wirtschaft mit ihren Rückwirkungen auf die Unternehmungen an.

Von 1960 bis 1975 baute Spanien eine moderne Industriestruktur ohne das entsprechende Management auf. Wirtschaftlichkeitsrechnungen waren bis vor kurzer Zeit noch unbekannt. Bis heute kann man beobachten, daß die spanische Unternehmensführung kaum mit Betriebswirtschaftlern besetzt ist. Im 
Rahmen des spanischen Industrialisierungsprozesses kann man in der Managemententwicklung drei Stufen feststellen.

In der ersten Phase betrifft die Sorge des Managements hauptsächlich die technischen und technologischen Betriebsprozesse.

In der zweiten Phase, am Ende der 60er Jahre, orientiert sich das Management auf den Absatzbereich, hauptsächlich auf nationaler Ebene. Das heißt, erst wenn die Produktionskapazitäten ausgebaut sind, beginnt die Sorge um den Absatz, bedingt durch die Entstehung starker Überkapazitäten. Eine internationale Orientierung ist nur in seltenen Fällen anzutreffen.

In der dritten Phase, mit Beginn der 70er Jahre, verstärkt sich in der Unternehmensführung die Sorge um die Organisation und das Personalwesen.

Insgesamt kann man feststellen, da $\beta$ das spanische Management bisher nicht unter der Herausforderung einer dynamischen und modernen Unternehmensführung gestanden hat. Es fehlte jeder Druck auf eine verbesserte Rationalität und auf eine angemessene persönliche und organisatorische Gestaltung der Führungsprozesse. Abgesehen von wenigen Ausnahmen hat die Unternehmensführung in Spanien bis heute keine Anpassungsprozesse in Hinsicht auf eine Wettbewerbswirtschaft vollzogen.

Dies stellt derzeit die größte Herausforderung dar. Die Schwierigkeiten bei der Durchsetzung einer marktwirtschaftlichen Ordnung stammen nicht nur von den Politikern und von der Verwaltung, die gegenüber Machtverlust empfindlich sind, sondern auch von seiten der Unternehmensführung selbst. Die Eingliederung der spanischen Wirtschaft in die EG weist hier eine der größten Schwierigkeiten auf, bedeutet aber gleichzeitig eine große Chance, die erforderliche Umstrukturierung der spanischen Wirtschaft voranzutreiben.

\section{Rahmen der Strategie der Spanischen Unternehmer}

In einer Wirtschaftsordnung, die durch einen traditionellen Interventionismus des Staates gestaltet ist, wo die Märkte auf einer sehr breit regulierten Struktur aufbauen, wo die Preise bei vielen Produkten festgelegt werden, wo von der Arbeitsmarktregelung eine betriebliche Anpassung an die neue Marktbedingungen unmöglich gemacht wird, wo auf den Finanz- und Kreditmärkten in sehr strenger Form interveniert wird, wo die Sanierung der öffentlichen Finanzen eine sehr dringende Aufgabe darstellt, kann ein rationaler Einsatz der Reserven nicht stattfinden.

Dazu muß man berücksichtigen, daß die Tendenzen über die Entwicklung und Änderung dieser Situation nicht eindeutig erscheinen. Heute ist eine Sanierung der öffentlichen, wie in vielen Fällen auch der privaten Unternehmen als Folge der ständigen Dekapitalisierung der Unternehmen notwendig. Dazu muß der starke Bedarf an Finanzierungsmitteln für die Rationalisierung und Modernisierung der Produktionsprozesse gerechnet werden.

In bezug auf Produktstruktur und -prozeß muß man die jahrelang fehlende Forschung und Entwicklung feststellen, so daß die Wettbewerbsfähigkeit der spanischen Industrie von dieser Seite keine Unterstützung bekommen hat. Aber eines der Hauptmerkmale ist, daß als Folge der jahrelang aufrechterhaltenen 
Wirtschaftsordnung ein modernes Management bei den spanischen Firmen fehlt. Die große Management- und Organisationslücke erscheint auch, und vielleicht besonders, bei den öffentlichen Unternehmen und bei der Verwaltung.

Im Prinzip kann man im Agrarsektor große Umstellungen auf Grund der Anwendung moderner Agrartechnologien durchführen, die die Landwirtschaft auf ein anderes Produktionsprogramm als heute ausrichten kann. Angesichts der großen Hindernisse in der Übergangszeit in die EG können die vorhandenen Überschüsse der Landwirtschaft viele Bereiche schwer belasten. In fast allen heute regulierten EG-Märkten kann die spanische Landwirtschaft eine sehr starke Anhebung der Produktivität erreichen. Spanischerseits muß gerade in diesen Bereichen mit sehr großen Anstrengungen gerechnet werden, um eine Flexibilität der Produktionsprogramme zu erreichen. Es wäre grundsätzlich falsch, die gegenwärtige Struktur des Agrarsektors einfach zu extrapolieren.

Welches die Produktionsprogramme bei der neuen internationalen Arbeitsteilung sein werden, ist eine offene Frage. Nur eine ausgedehnte Unternehmerschicht kann im Laufe der Anpassung eine Antwort geben.

In bezug auf die Industrie ist heute praktisch jegliche Prognose sicher falsch. Die gegenwärtige Gestaltung der Märkte, die direkten und indirekten bzw. verschleierten Subventionen und wettbewerbsbeschränkenden Maßnahmen erlauben heute nicht, eine Prognose über die Wettbewerbsfähigkeiten zu stellen.

Im Prinzip kann man heute eine sehr eindeutige Dualität in der spanischen Wirtschaft erkennen.

Einerseits gibt es die multinationalen Firmen jüngeren Gründungsdatums, die die Anpassungszwänge schon bei der Gründung berücksichtigt haben. Diese Firmen sind durch eine moderne sehr stark rationalisierte Produktionsanlage, durch eine im Vergleich bessere Kapitalstruktur, durch ausgereifte Produkte und Prozesse sowie durch eine entwickelte Marktstruktur und ein modernes Management gekennzeichnet.

Gegenüber diesen leistungsfähigen Firmen, die in der neuen internationalen Arbeitsteilung von vornherein eingebettet sind, stehen die meisten der spanischen Firmen und Multinationalen älteren Gründungsdatums. Diese Unternehmen sind mit sehr veralteten Produktionsanlagen ausgerüstet und haben einen starren und zu großen Personalbestand. Die Rationalisierungsprozesse werden nur auf Kosten der Lebensfähigkeit der Firmen durchgeführt. Es handelt sich um Firmen mit einem zu hohen Personalbestand, einem sehr geringen Eigenkapital, um stark verschuldete Unternehmen mit einer sehr geringen Anpassungskapazität.

Diese Dualität bringt gleichzeitig eine Reihe schwieriger Interdependenzen zwischen beiden Gattungen von Firmen mit sich. Die ,alten“ werden noch weiter belastet und eine Modernisierung und Sanierung praktisch unmöglich gemacht. Die ,neuen“ bekommen wieder staatliche Genehmigungen, Subventionen, Privilegien, die wiederum die Produktionsprozesse und die Märkte weiter verzerren.

Gerade im Industriesektor wirkt sich vor allem dieser eingefleischte Interventionismus des Staates aus. Obwohl die Erfahrungen der fünfziger und sechziger Jahre negativ zu bewerten sind, wiederholt man heute dieselbe Prozedur. 
Das Industrieministerium weiß besser als der Markt und die Unternehmer, wie viele Videomarken in Spanien hergestellt werden müssen. Diese ministerielle Überzeugung der Fähigkeit der Funktionäre für das Aufspüren von Märkten, Produkten und Prozessen ist unglaublich. Die Sektoren, die jetzt ,restrukturiert" werden, sind alles Sektoren, die vor zwanzig Jahren eine Genehmigung haben mußten. Die sich wiederholenden Zyklen müssen abgebrochen werden. Dafür braucht man sowohl Unternehmer und Politiker, aber auch eine Gesellschaft, die positiver gegenüber der Unternehmeraktivität steht.

Im Dienstleistungssektor muß man einerseits die Fremdenverkehrsposition Spaniens und andererseits und vor allem die Finanzinstitutionen untersuchen. Diese letzten Institutionen werden in der Übergangszeit einem sehr entscheidenden Anpassungsdruck ausgesetzt werden, da die heutige Situation im Vergleich zu den EG-Ländern wie folgt charakterisiert werden kann:

1. Die Betriebsgröße der Finanzinstitution ist sehr klein.

2. Die Filialenstruktur ist zu weit ausgedehnt. Spanien hatte 198215500 Bankfilialen gegenüber 5000 in der BRD.

3. Höhere Transformationskosten.

4. Sehr geringere Internationalisierung der Banktätigkeit.

In bezug auf den Fremdenverkehr muß nach einer sehr expansiven Periode sowohl eine Modernisierung der Anlagen und Organisationsformen durchgeführt werden, als auch ein besseres Marketing bei einer höheren qualitativen Leistung. Die vorhandene Struktur und Organisation muß renoviert werden und auf ein höheres Differenzierungsprogramm eingehen.

Eine Änderung der Wirtschaftsordnung Spaniens kann eine Wendung im Verhalten und in der Organisation entwickeln, so daß eine Modernisierung des Landes in kurzer Zeit stattfinden kann. Der Druck des Wettbewerbs und eine entsprechende Information der Spanier über neue Organisationsformen können ganz positive Impulse entwickeln. Land.

Information und Ausbildung sind die entscheidenden Signale für dieses

Die heutige ordnungspolitische Situation Spaniens muß man vor dem Hintergrund des traditionellen Interventionismus des Staates sehen. Die administrativen Regelungen in bezug auf eine sehr umfangreiche Preisfestsetzung, administrative Verfahrensregelungen in Produktionsprozessen, die staatliche Regulierung der Lohnfindung und die umfangreiche Normierung im Arbeitsrecht, die fehlende Konvertibilität der Peseta und die tiefgreifenden Interventionen im Devisenverkehr, die heute bestehende Lenkung der Passivbestände der Sparkassen und Privatbanken ( $2 / 3$ der Sparkassen und über $1 / 2$ der Banken werden durch staatliche Maßnahmen gelenkt), u. a. m. bestimmen die gegenwärtige spanische Wirtschaftsordnung: D. h., eine verwaltete Wirtschaft nach rein diskretionellen Kriterien.

Es fehlt an Flexibilität für die Anpassung der Unternehmen, es fehlt an eigener Verantwortung für Risikoübernahme. Die Unternehmen sind nicht fähig, die notwendige Anpassung von Personal- und Kapitalstrukturen an die neuen Märkte und Technologien durchzuführen. 
In Spanien geht es mit dem Beitritt in die EG vom 1. Januar 1986 nicht nur um eine reine wettbewerbsmäßige Anpassung seiner Wirtschaft und seiner Unternehmen, sondern es handelt sich vor allem um eine Umwandlung von Institutionen, Normen, Verhalten und Werten, die einen tief in der spanischen Gesellschaft inkrustierten Staatsinterventionismus ablösen muß. D. h. die Setzung von ganz anderen Maßstäben, die die gegenwärtige Strategie ersetzen muß. Das bedeutet aber vor allem eine neue Unternehmenskultur. Dies kann auf keinen Fall durch eine sogenannte pragmatische Anpassung erfolgen, sondern es muß durch eine energische und eindeutige Stellungnahme des Staates für eine marktwirtschaftliche Ordnung geschehen. Es handelt sich um eine deutliche Festlegung seitens des Staates, daß die Wirtschaftsprozesse Risiken und Chancen anbieten, die von Unternehmen übernommen werden müssen.

Man muß von einer Wirtschaftsstruktur Abstand nehmen, wo viele von den Unternehmern gute Verwalter sind, aber keine Unternehmer. Die ständige Intervention des Staates im Unternehmen selbst durch Festsetzung der Allokation der Ressourcen im Betrieb, durch die Regulierung der Märkte und die Förderung von Monopolen bzw. Quasi-Monopolen und kartellartigen Unternehmenskonzentrationen hat einen Typus von Unternehmer bzw. Manager entwickelt, der gerade gegenüber einer wettbewerbsfördernden Wirtschaftsordnung ein sehr großes Anpassungsproblem zeigt. Dies trifft auch auf Politiker und Beamte zu.

Bei einer vor kurzer Zeit durchgeführten Umfrage in Unternehmerkreisen darüber, welche Wirtschaftsordnung sie als angebracht für die Unternehmenstätigkeit wählen würden, sprachen sich $77 \%$ für eine freie Marktwirtschaft und $10,5 \%$ für eine soziale Marktwirtschaft aus, d. h. $89 \%$ sind für eine marktwirtschaftliche Ordnung, vor allem bei mittleren und kleinen Unternehmen. Aber gleichzeitig waren nur $31 \%$ für freie Spielregeln ohne staatliche Intervention, während $50 \%$ für die Anwendung einer indikativen Planung, für Subventionen, etc. als ordnungspolitische Gestaltungsmaßnahmen plädieren. D. h., man will eine freiheitliche Wirtschaftsordnung, aber der Staat muß die Rahmen der Unternehmenstätigkeit festlegen, um weiterhin mit einem besonders privilegierten Interventionismus die Koordinierung der Wirtschaft durchzuführen.

Das fehlende Vertrauen in die Regulierungsfunktionen des Marktes, die unzureichende Auffassung und Instrumentierung des ,sozialen Ausgleichs“ und die fehlende Modernisierung des Managements, sind die Merkmale dieser widersprüchlichen Stellungnahme der spanischen Unternehmerschaft. Aber auch ähnlich wird dies in Gewerkschaftskreisen, und zwar noch schärfer akzentuiert, verlangt.

Nur durch eine gezielte Wettbewerbsordnung und das Zurückziehen des Staates von der Wirtschaft in der gegenwärtigen Form bei der Allokation der Ressourcen, besteht die Möglichkeit, diese gewaltige Umwandlung der spanischen Gesellschaft durchzuführen. Diese ist heute, nach der oben zitierten Umfrage, nur mit $21 \%$ für eine Wirtschaftsordnung, die auf privatem Eigentum basiert, während $22 \%$ für Selbstverwaltung und $33 \%$ für eine wirtschaftliche Mitbestimmung sind.

Sowohl von den sozialen Partnern wie auch von der Öffentlichkeit, den Parteien und der Gesellschaft muß immer noch eine gewaltige Überzeugungs- 
arbeit über die Vorteile einer sozialen marktwirtschaftlichen Ordnung durchgeführt werden.

Man muß auf die Festlegung neuer Rahmenbedingungen für die Unternehmenstätigkeit bestehen. Die kulturelle Herausforderung an die spanischen Manager ist gewaltig.

\section{Auswirkungen der Wirtschaftsordnung auf die Unternehmens- führung}

Für die Entwicklung einer dynamischen und leistungsfähigen Unternehmensführung stellt eine liberale Wirtschaftsordnung die wichtigste Grundlage dar. Jede interventionistische, verwaltende Wirtschaftsordnung führt zu einer statischen Unternehmensführung, da die Anpassung an die Umwelt keine Herausforderung mehr darstellt. Diese Hypothese kann an der spanischen Entwicklung belegt werden.

Die bremsende Wirkung einer verwaltenden Wirtschaftsordnung ist sowohl die Konsequenz des fehlenden Wettbewerbs, wie auch die Folge der Verlagerung der echten Führungsentscheidungen von der Unternehmensebene auf die überbetriebliche Ebene, d. h. auf staatliche Verwaltungsinstanzen. Entscheidungen über die Preisgestaltung oder die Finanzierungsstrukturen gehören zu den Entscheidungsbereichen, die auf überbetriebliche Ebenen verlagert werden. Auf Unternehmensebene bleiben nunmehr Verwaltungsaufgaben und die technische Leitung. Bei dieser Wirtschaftsordnung bestehen keine Möglichkeiten für die Entwicklung einer modernen Unternehmensführung.

Die Betonung des dispositiven Faktors gegenüber den elementaren Faktoren hat schon seit Gutenberg in der betriebswirtschaftlichen Theorie eine systematische Behandlung erfahren. Die Entwicklung der Managementlehre hat in den letzten zwei Jahrzehnten Grundlagen geliefert, so daß wir heute in der Lage sind, das Wirtschaftspotential eines Landes am Know-how des Managements zu messen. Hier müssen die Chancen eines jeden Landes entwickelt werden.

In diesem Sinne haben wir versucht, empirisch die Beziehungen zwischen Unternehmen und Unternehmern mit der Umwelt zu ermitteln. Dazu haben wir in einer ersten Annäherung die Führungs- und Organisationsstrukturen der spanischen Unternehmen analysiert.

Als Ergebnis dieser Untersuchung lassen sich folgende Thesen formulieren:

1. Als Folge der verwaltenden Wirtschaftsordnung und des fehlenden Wettbewerbs entspricht die spanische Unternehmensführung nicht der technischen und technologischen Entwicklung.

2. Heute fehlt immer noch jeder dynamische Druck; und es fehlt die Förderung der notwendigen wirtschaftspolitischen Bedingungen.

3. Die Ausbildungsinstitutionen auf jedem Niveau sind heute noch sehr weit davon entfernt, in der Ausbildung und in der Lehrtätigkeit den Erfordernissen eines modernen Managements zu entsprechen. 
4. Von der Unternehmensseite her hat sich eine positive Entwicklung durchgesetzt: die mit Führungsaufgaben beauftragen Manager sind heute bereit, die Anpassung durch Lernprozesse mitzumachen.

5. Eine der Hauptbedingungen für die Anpassung der spanischen Wirtschaft hängt von der Managementausbildung ab. Dabei müssen drei Aufgaben erfüllt werden:

a) Ausbildungsprogramme

b) Reformen der Ausbildungsstruktur an den Universitäten und Technischen Hochschulen

c) Umdenken der spanischen Unternehmen bezüglich der Rolle des Unternehmers in einer pluralistischen Wirtschaftsordnung.

6. Die Eingliederung in die EG bedeutet ein sehr tiefe Umwandlung nicht nur der spanischen Produktions- und Institutionsstruktur, sondern vor allem im Management.

Die großen Chancen des Beitritts Spaniens zur EG liegen heute in der Möglichkeit der Gestaltung einer Wirtschaftsordnung, die durch den Wettbewerb und den entsprechenden sozialen Ausgleich harmonisch gestaltet wird, nämlich:

1. Durch den Beitritt muß Spanien in vielen Bereichen des Rechts eine Modernisierung der Normen und des Verhaltens mit sehr geringen Kosten und in kurzer Zeit durchführen.

2. Durch den Beitritt kann eine wettbewerbsorientierte Wirtschaftsordnung mit geringerem Widerstand eingeführt werden.

3. Durch den Beitritt wird sicher eine neue Generation von Unternehmern und Führungskräften heranwachsen, die die Anpassung Spaniens schneller, als man heute denkt, durchsetzen wird.

4. Im Hinblick auf den Beitritt werden auch die Gewerkschaften an die Festlegung einer neuen Gewerkschaftspolitik denken müssen.

5. Durch den Beitritt muß die Regierung und die öffentliche Verwaltung auf die Bindung einer harmonischen Wirtschaftspolitik mehr achtgeben.

6. Der große Beitrag der EG für Spanien bedeutet den Eintritt in eine Modernisierung seines Produktionsprozesses, seiner wirtschaftlichen Normen, seiner Wirtschaftseinstellungen und die Freilegung von unternehmerischen Kräften, die sofort wirken können.

Nur durch eine marktwirtschaftliche Ordnung kann Spanien in sehr kurzer Zeit den großen Sprung der Anpassung durchführen.

Zum ersten Mal kann von der Gestaltung einer Wirtschaftsverfassung gesprochen werden und wird an die Festlegung einer neuen Gewerkschaftspolitik gedacht werden müssen.

Schwächen zeigen sich auch in der Ordnung der Entscheidungsmächte in der Unternehmung. Nur zwei bis drei Personen haben faktische Entscheidungsmacht: Das bedeutet, daß die Führungsspitze sehr eng ist. Die Wirkung dieser Struktur drückt sich hauptsächlich in der sehr geringen Mitwirkung der anderen Manager an den Entscheidungsprozessen aus. Dieser Prozeß ist eine Folge der fehlenden Festlegung der juristischen Verantwortung der Manager. 
Bei einer weiteren Analyse der Ergebnisse kann man feststellen, daß

1. in den spanischen Unternehmen seit kurzer Zeit eine große Sorge um die Führungsprobleme existiert,

2. der spanische Manager heute bereit ist, neue Ansätze und Organisationstechniken zu lernen und anzuwenden,

3. der spanische Manager neue Informationen über diese Ansätze sucht; er ist aber äußerst vorsichtig gegenüber eventuellen Mißerfolgen bei der Anwendung. Die Erfahrungen in den sechziger und siebziger Jahren geben Anlaß zur Vorsicht in Managementfragen.

Diese Entwicklung bestätigt die oben aufgestellte Hypothese, daß man Impulse für eine dynamische und anpassungsfähige Unternehmensführung nur erwarten kann, wenn die Wirtschaftsordnung eine Öffnung des Entscheidungsprozesses fördert.

In bezug auf die Existenz einer strategischen Führung überrascht die hohe positive Zahl von Antworten ( $58 \%$ ), und es erweckt großes Interesse, daß $80 \%$ der Befragten die Notwendigkeit einer Unternehmensstrategie bejahen. Die Unternehmer sind also bereit, die Technik der strategischen Führung anzuwenden, wenn sie über die Kenntnisse und die notwendigen Grundlagen verfügen. Dieses Interesse an strategischer Führung bei den spanischen Unternehmen ist Ausdruck des verlorenen Vertrauens in die bisher angewandten Führungsgrundsätze.

Eine sehr aufschlußreiche Angabe ist der kurzfristige Planungshorizont: ein Jahr. Dieser Tatbestand ist ein Beweis für die bisher fehlende strategische Führung.

Bei der Analyse der Antworten bezüglich der Existenz von Zielsystemen als Instrument der Unternehmensführung kann man feststellen, daß 95\% der Großunternehmen und $82 \%$ der mittleren Betriebe über solche Systeme verfügen. Allerdings drückt dieser hohe Anteil oft mehr einen Wunsch als eine Realität aus. Nur bei einer geringen Anzahl der befragten Unternehmungen liegen diese Ziele schriftlich vor, wobei es sich immer lediglich um Jahresziele handelt. Das heißt, es liegen keine formalisierten Zielsysteme vor, und es handelt sich mehr um Budgetangaben als um Unternehmens- bzw. Bereichsziele, die als echte Führungsinstrumente angewandt werden. Auch bei großen Unternehmen bedeutet ein Zielhorizont von drei Jahren nicht gleichzeitig die Anwendung eines Zielsystems als Führungsinstrument.

Sowohl bei der Analyse der Strategie-Anwendung in der Unternehmensführung als auch bei den Zielsystemen und der Organisationsstruktur kann man eine starke Zentralisierung feststellen. Die sehr schwache Mitarbeit an der Entscheidungsfindung in der Führungsstruktur stellt eine der größten Schwächen der spanischen Unternehmen dar.

Ein interessantes Ergebnis der Untersuchung ist das Interesse an Führungsstilen, obwohl diese in der Praxis der Unternehmensführung bisher kaum zur Anwendung gelangen. Die Möglichkeiten der Anwendung von Führungsstilen als Koordinationsinstrument werden deutlich gesehen.

Was die Organisationsstrukturen anbetrifft, so verfügen nur $34 \%$ der Unternehmungen über ein Organisationsschema und eine Funktionsbeschreibung. 
Der Bereich der Organisation stellt eine der Schwachstellen der spanischen Unternehmungen dar.

Die stark akzentuierte Zentralisierung der Unternehmungsführung bedeutet eine niedrige Bewertung des Organisationsbedarfs und fördert eine mehr informelle Organisationsstruktur. Es gibt auch heute noch Präferenzen in der spanischen Unternehmensführung für eine informelle, persönliche und flexible Handhabung der Organisationsstruktur mit einer ständigen neuen Verteilung von Macht und Funktionen. Dies führt dazu, daß eindeutige Aufgabenstrukturen fehlen und daß das mittlere Management kaum an den Entscheidungsprozessen beteiligt ist.

Diese Situation wird auch von den Befragten gesehen, und deshalb sind $56 \%$ der Meinung, daß eine Dezentralisierung der Unternehmensführung stattfinden sollte.

An letzter Stelle wurde die Problematik der Informationssysteme behandelt. In diesem Bereich sind die traditionellen Informationssysteme der Finanzbuchhaltung praktisch dominierend. Wenn man bedenkt, daß diese Informationssysteme bei der Bindung an die Steuerbilanz und bei den hohen Inflationsraten kein geeignetes Informationsinstrument darstellen, kann man feststellen, daß eine große Lücke in diesem Bereich der Unternehmensführung besteht. Die Kostenrechnung, die ebenfalls mehr an die Finanz- und Steuerbuchhaltung als an die technischen und kaufmännischen Prozesse gebunden ist, und andere moderne Informationssysteme sind nur schwach vertreten.

Man erhält bei $70 \%$ der Manager die Antwort, daß sie mehr über Informationssysteme wissen wollen. Trotzdem sind sie sich heute noch nicht im klaren, welche Bedeutung den Informationssystemen für die Entscheidungsprozesse zukommt.

Zusammenfassend kann man folgendes feststellen:

1. Es besteht eine sehr starke Zentralisation bei der Unternehmensführung.

2. Es besteht eine deutliche Bereitschaft zur Modernisierung und Anpassung bei der spanischen Unternehmensführung.

3. Die Manager wissen, daß eine Änderung in den Führungsstilen stattfinden muß und daß dies mehr Mitwirkung erfordert.

4. Im Bereich der Organisation und der Informationssysteme ist die Vernachlässigung wesentlich größer als auf den übrigen Gebieten.

5. Die Verwirklichung einer strategischen Führung stellt noch ein großes Fragezeichen für die Manager dar.

6. Ohne eine entsprechende Stabilisierung und Ausformulierung der Wirtschaftsordnung wird eine Erweiterung des Planungshorizontes und die Einführung eines leistungsfähigen Rechnungswesens nur schwer zu erreichen sein.

\section{Bewertung der spanischen Situation}

1. Der Industrialisierungsproze $ß$ ist im Rahmen einer verwalteten Wirtschaft durchgeführt worden. Dies hat zu einer Disharmonie zwischen der techni- 
schen und technologischen Entwicklung einerseits und der Management-Entwicklung andererseits geführt.

2. Im Vergleich mit der Entwicklung in anderen Bereichen der Unternehmungen ergibt sich ein Zurückbleiben des Managementniveaus um mehr als 15 Jahre.

3. Dies entspricht dem Zurückbleiben der spanischen Universitäten im Hinblick auf betriebswirtschaftliche Forschung und Lehre.

4. Die Managementausbildungszentren sind nur ungenügend entwickelt worden.

5. Die Herausforderung durch eine dynamische Wirtschaftsordnung bringt folgende Konsequenzen mit sich: Es müssen

- insgesamt Anpassungsprozesse schneller vorangetrieben werden,

- eine Anpassung der Ausbildungsprogramme erfolgen,

- Prozesse der Auswahl der besten Personen für die Managementfunktionen entwickelt werden,

- schnelle Anpassungsmöglichkeiten an die sich ändernde Umwelt geschaffen werden,

- schnellere Reaktionen auf sich verändernde ökonomische und gesellschaftliche Prozesse erfolgen.

6. In einer interventionistischen Wirtschaftsordnung sind die Möglichkeiten, eine dynamische Unternehmensführung zu fördern, begrenzt, da die Herausforderung an die Leistung des Managers sehr schwach ist.

7. Als Folge des fehlenden Management-Know-hows ist Spanien in einer ungünstigeren Lage als andere Länder, um einen Ausweg aus der aktuellen Wirtschaftskrise zu finden.

\section{Die strategische Anpassung der spanischen Unternehmen}

Die erwähnte Entwicklung des spanischen Managements führt zu einer der härtesten Konfrontationen bei der Einfügung der spanischen Wirtschaft in die internationale Arbeitsteilung.

Das spanische Management hat nur eine ganz deutliche Strategie durchgeführt:

Eine Produktionsstruktur, die hauptsächlich an regulierten Märkten orientiert ist. Die Anpassungsbedürfnisse sind während längeren Zeiträumen durchgeführt worden und durch die Regulierung der Rahmenbedingungen brauchte man kaum über Anpassungsflexibilität zu verfügen.

Das spanische Unternehmen hat praktisch keine Strategie im Sinne der Ausrichtung auf Märkten und Kunden, sondern es ist hauptsächlich am Rahmen der vom Staat festgelegten Handlungen orientiert.

Es geht in der Realität um eine reine Verwaltungsfunktion eher als um eine Unternehmerfunktion. Abgesehen von wenigen Ausnahmen kann man heute von einer sehr schlechten Integration des Menschen im Unternehmen sprechen. 
Die Strategie der spanischen Unternehmen kann man zusammenfassend wie folgt darlegen:

1. Als Folge der stabilen Elemente der Umwelt besteht eine kurzfristige Anpassungsstrategie in allen Bereichen der Unternehmen. Die Prämisse der Stabilität der Umwelt ersetzt eine langfristige Strategie bei den Unternehmen.

2. Man hat hauptsächlich eine Strategie auf den nationalen Märkten. Die ausländischen Märkte sind eher subsidiärer bzw. konjunktureller Art. Es fehlt deshalb eine Außenhandelsorganisation.

3. Im Personal-, Finanz- und Einkaufsbereich wenden die spanischen Unternehmen eine kurzfristige Anpassungsstrategie immer in der Hoffnung an, Mengen und Preise zu festgelegten Bedingungen zu erhalten. Deshalb besteht eine nur kurzfristige Disposition.

4. Infolge der hohen Bedeutung des Inlandsmarktes hat man auch eher innerhalb kartellartiger Bedingungen eine kurzfristige Anpassungsstrategie ausgewählt.

5. Kontrollfragen werden immer nur im technischen Bereich erkannt. In der Haushaltsmaschinenindustrie hat man die Kontrollkosten bis auf $16 \%$ der gesamten Produktionskosten geschätzt (Japan ungefähr 4\%).

6. Die Folge dieser kurzfristigen Anpassungsstrategie an eine „stabile“ Umwelt hat folgende Konsequenzen gebracht:

a) Niedrige Ausnutzung der Kapazitäten (ca. 60/70\%)

b) Niedrige Produktivität ( $56 \%$ des EG-Durchschnitts)

c) zu hohe Produktionskosten

d) niedrige Qualität

e) starke Verschuldung der Unternehmen

f) fehlende langfristige Personalpolitik bzw. nur eine verwaltete Personalwirtschaft

g) fehlende Forschung und Entwicklung

h) Management als Verwaltung interpretiert

7. Im Augenblick des Bruches in der „stabilen Umwelt“ (ab 1975) sind die spanischen Unternehmen nicht mehr fähig, als Institution und Management die Anpassung durchzuführen. Es gibt heute nur eine Strategie des Überlebens.

In bezug auf die Unternehmenskultur kann man folgende Bewertung vornehmen:

a) Die patriarchalische Führungspolitik der 50er Jahre und die Immobilität des Managements hat man bis heute durch keine neue Unternehmenskultur ersetzt.

b) Es fehlt an der Definition einer neuen Unternehmenskultur. Die fehlende Unternehmensphilosophie und -politik hat eine solche Änderung nicht hervorgerufen. Die „Tagesanpassungssorge“ läßt keinen Spielraum für diese grundsätzlichen Fragen.

c) Die fehlende Kultur provoziert eine sehr mangelhafte Integration und Identifizierung von Manager und Personal mit den Firmen. Die Situation einer „Übergangslage“" schadet der Anpassung der spanischen Unternehmen. 
d) Solange keine bewußte Unternehmenskultur entwickelt wird, wird es schwerfallen, eine Wirtschaftsstrategie durchzuführen.

e) Die Hauptaufgabe beim heutigen Management ist die bewußte Integration von Unternehmenskultur und -strategie in Denken und Handeln. Ohne diesen Schritt wird die notwendige Festlegung von Unternehmenspolitik und die dringend notwendige neue Organisationsgestaltung nur kurzfristiger Natur sein. 